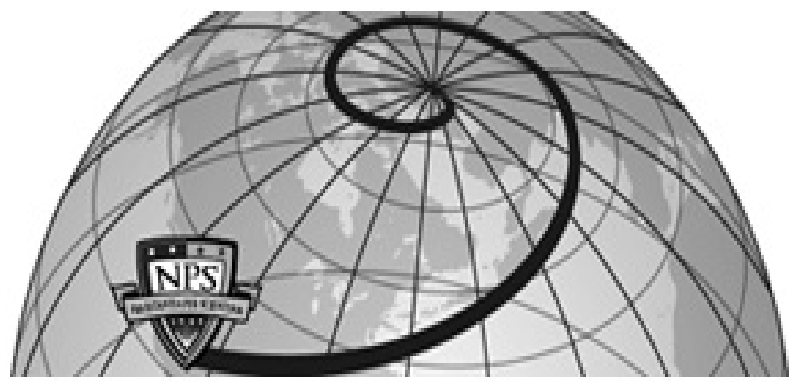

Calhoun: The NPS Institutional Archive DSpace Repository

\title{
Analytical solutions for the ACC and its overturning circulation
}

\section{Radko, Timour}

Journal of Marine Research, 63, 10411055, 2005

https://hdl.handle.net/10945/42124

This publication is a work of the U.S. Government as defined in Title 17, United States Code, Section 101. Copyright protection is not available for this work in the United States.

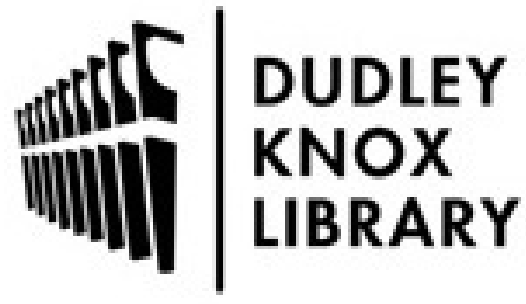

http://www.nps.edu/library
Calhoun is the Naval Postgraduate School's public access digital repository for research materials and institutional publications created by the NPS community. Calhoun is named for Professor of Mathematics Guy K. Calhoun, NPS's first appointed -- and published -- scholarly author.

Dudley Knox Library / Naval Postgraduate School 411 Dyer Road / 1 University Circle Monterey, California USA 93943 


\title{
Analytical solutions for the ACC and its overturning circulation
}

\author{
by Timour Radko ${ }^{1}$
}

\begin{abstract}
An explicit analytical model of the Antarctic Circumpolar Current (ACC) is presented in which the key feature is the balance between the Eulerian circulation acting to overturn the isopycnals and geostrophic eddies which tend to flatten them. Solutions for the stratification and overturning circulation are obtained by expanding the governing residual mean equations of motion in a small parameter $(\varepsilon)$ which measures the relative strength of the surface buoyancy flux and mechanical forcing by winds. Our balanced asymptotic model extends and reconciles the earlier views on the dynamics of the (streamwise-averaged) ACC by demonstrating that the zero order balance between the eddy-induced circulation and the mean flow determines gross features of the buoyancy distribution in the interior of the ACC. The diabatic buoyancy fluxes are essential in driving the secondary (residual) circulation which is, however, largely steered along the pathways set by the dominant adiabatic balance. Model assumptions are supported by a close agreement of the analytical solutions and fully nonlinear numerical calculations.
\end{abstract}

\section{Introduction}

Much of the modern perception of the dynamics of the Antarctic Circumpolar Current system (ACC) is based on the idea of a competition between the two mechanisms of meridional transport - the Eulerian circulation and eddy-induced transfer. The schematic diagram in Figure 1 (from Marshall and Radko, 2003, MR hereafter) illustrates the tendency of the time mean flow $(\bar{\Psi})$ to overturn the isopycnal surfaces $(\bar{b})$ and geostrophic eddies $\left(\Psi^{*}\right)$ tending to flatten them out. The approximate cancellation of the eddy-induced and Eulerian circulation, often referred to as vanishing of the Deacon cell, is a prominent feature of numerical simulations (e.g. Danabasoglu et al., 1994). The relatively weak residual flow is represented by a streamfunction

$$
\Psi_{r e s}=\bar{\Psi}+\Psi^{*}
$$

which advects buoyancy and passive tracers in the meridional plane to offset the diabatic sources and sinks.

The assumed balance between $\bar{\Psi}$, which is set by the wind stress, and $\Psi^{*}$ (set by eddies)

1. Department of Oceanography, Naval Postgraduate School, Monterey, California, 93943, U.S.A. email: tradko@nps.edu 


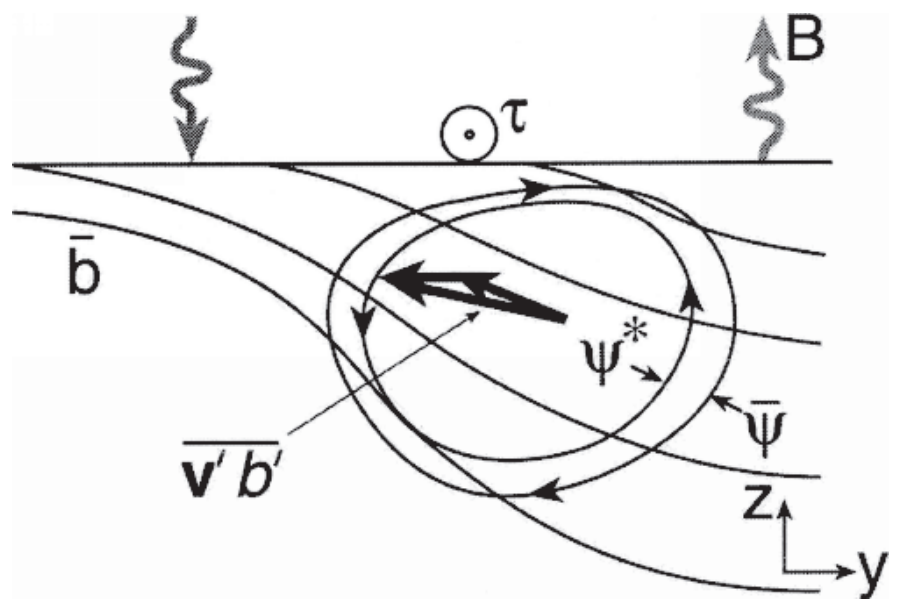

Figure 1. Schematic diagram of the Eulerian mean circulation $(\bar{\Psi})$, which tends to overturn the isopycnal surfaces, and the eddy-induced transport ( $\left.\Psi^{*}\right)$ acting in the opposite sense (from MR).

combined with a closure for the eddy buoyancy transfer $\left(\overline{v^{\prime} b^{\prime}}\right)$, made it possible to formulate a theory for the zonal transport of the ACC (Johnson and Bryden, 1989). MR went further and argued that the competition between eddies and the mean flow is also essential to understanding the stratification and meridional overturning of the ACC. The general view of the importance of mesoscale eddies in the (quasi)-zonal flows finds support in the numerical (Karsten et al., 2002) and laboratory (Cenedese et al., 2004) studies. However, specific mechanisms of interaction between the wind forcing, air-sea fluxes and eddy transport in the interior, and their respective roles in controlling the patterns of buoyancy and residual circulation, are still poorly understood (Bryden and Cunningham, 2003). For instance, upwelling of the upper circumpolar deep water and equatorward flow at the surface apparently requires buoyancy gain; Speer et al. (2000) examined the air-sea fluxes and concluded that the air-sea fluxes are compatible with the mass transports in the Southern Ocean. However the question remains (e.g., Hallberg and Gnanadesikan, 2001) whether the surface buoyancy fluxes and/or small-scale mixing processes play largely passive roles in maintaining the overturning circulation of the ACC, or whether they can actively participate in setting up its density structure. While these fundamentally diabatic effects (and hence the associated residual circulation) are often neglected in the extant theories for the structure and transport of the ACC, their a priori exclusion lacks physical grounds (Olbers et al., 2004).

Although a considerable effort has gone into the realistic modeling studies of the ACC, these simulations still do not provide definite answers as to the roles of the mechanical and thermodynamic forcing in shaping up the ACC. Numerical results are known to be sensitive to the model formulations; for instance, values of the zonal transport in models are spread over a range from well under $100 \mathrm{~Sv}$ to well over $200 \mathrm{~Sv}$ (Olbers et al., 2004). 
While most studies reveal the critical role of the wind stress in controlling the stratification and transport of the ACC, significance of the buoyancy forcing is much less certain. Doos (1994) modeled the meridional circulation without any buoyancy forcing and found results similar to the numerical simulations of Doos and Webb (1994), whereas Gnanadesikan and Hallberg (2000) argued that the buoyancy forcing could play a role in setting up the density structure. Furthermore, the complexity of the realistic numerical models makes the dynamical interpretation of results extremely difficult, particularly with regard to the "higher order" diabatic effects discussed in this note.

Our goal is to help unravel the details of interplay between the distinct dynamical components of the ACC by developing the analytical weak residual circulation theory of the ACC based on the asymptotic limit

$$
\varepsilon=\frac{\left\langle\Psi_{r e s}\right\rangle}{\langle\bar{\Psi}\rangle} \ll 1
$$

where $\left\langle\Psi_{\text {res }}\right\rangle$ is the scale of the residual circulation averaged along the path of the ACC, and $\langle\bar{\Psi}\rangle$ is the scale of the mean streamfunction - the Ekman circulation in the meridional plane. We argue that this limit adequately represents the parameter range of the real circumpolar current. The leading order balance of the governing residual mean equations of motion therefore reduces (1) to the balance between the mean circulation and eddyinduced flow - vanishing of the Deacon cell - whereas the second order balance explicitly describes the dynamics of the residual circulation and its relation to the surface buoyancy fluxes. Basic physics and dynamics at play are illustrated by constructing explicit analytical solutions of the model equations and comparing them with the fully nonlinear results.

\section{Theoretical framework}

Following MR, we consider the model of the ACC in which we reference our along-stream coordinate $x$ to a mean surface geostrophic contour; $y$ is the coordinate normal to the stream. Eddy fluxes normal to these contours are then by construction due to transient rather than standing eddies.

It has been long recognized (Andrews and McIntyre, 1976) that the distribution of buoyancy and tracers in the eddying flows cannot be accounted for by the Eulerian mean circulation, but also involves the eddy-induced advection, a process similar to the Stokes drift. It is assumed that the eddy transport is largely directed along the time mean buoyancy surfaces (the limit of "adiabatic eddies"), and therefore

$$
\overline{\overline{w^{\prime} b^{\prime}}}=-\bar{b}_{y},
$$

where $\left(\overline{v^{\prime} b^{\prime}}, \overline{w^{\prime} b^{\prime}}\right)$ are the eddy buoyancy flux components; averages are taken in time and 
along the streamlines. The eddy streamfunction in this case can be defined (Held and Schneider, 1999) as

$$
\Psi^{*}=-\frac{\overline{w^{\prime} b^{\prime}}}{\bar{b}_{y}}=\frac{\overline{v^{\prime} b^{\prime}}}{\bar{b}_{z}} .
$$

Introducing the eddy streamfunction (4) greatly simplifies the time-mean streamline averaged buoyancy equation

$$
\bar{v} \frac{\partial \bar{b}}{\partial y}+\bar{w} \frac{\partial \bar{b}}{\partial z}=J(\bar{\Psi}, \bar{b})=-\frac{\partial}{\partial y}\left(\overline{v^{\prime} b^{\prime}}\right)-\frac{\partial}{\partial z}\left(\overline{w^{\prime} b^{\prime}}\right)+\frac{\partial B}{\partial z},
$$

where buoyancy forcing from the air-sea interaction and small-scale mixing processes are expressed as the divergence of a buoyancy flux $B$, and $J(a, b)=a_{y} b_{z}-a_{z} b_{y}$. For adiabatic eddies satisfying (3), buoyancy equation reduces to

$$
J\left(\Psi_{r e s}, \bar{b}\right)=\frac{\partial B}{\partial z},
$$

where $\Psi_{r e s}$ is the residual streamfunction in Eq. (1). Eq. (5) can be interpreted as a statement that the advection of buoyancy (and other tracers) on long time scales in the eddying field is accomplished by the residual, rather than mean, circulation.

If we assume that the eddies transfer properties down the large-scale gradient, the lateral eddy buoyancy flux can be (conventionally) expressed thus

$$
\overline{v^{\prime} b^{\prime}}=-K \bar{b}_{y}
$$

where the eddy transfer coefficient $K$ is not necessarily uniform but rather depends on the local characteristics of the flow. MR used a model in which $K$ is itself proportional to the isopycnal slope $\left(s=-\frac{\bar{b}_{y}}{\bar{b}_{z}}\right)$ :

$$
K=k_{0}|s|
$$

This relation was suggested by the numerical simulations in Visbeck et al. (1997) and by the simple scaling arguments (MR); it also finds qualitative support in studies of the laboratory analogue of the ACC by Cenedese et al. (2004). One can question the specific form of the eddy closure assumed, and so we describe and analyze an alternative parameterization in the Appendix. Differences and similarities between the two models can be used to separate the relatively robust features of the resulting solutions from the closure-dependent ones.

Our governing equations consist of the buoyancy equation (5), the time-mean Ekman transport below the Ekman layer:

$$
\bar{\Psi}=-\frac{\tau_{\rho}}{f}, \quad \tau_{\rho}=\bar{\tau} / \rho_{0}
$$


and the eddy parameterization (6), (7), which reduce the eddy streamfunction to

$$
\Psi^{*}=\frac{\overline{v^{\prime} b^{\prime}}}{\bar{b}_{z}}=K s=-k_{0} s^{2} .
$$

Following MR, the problem is solved separately in a thin, vertically homogeneous mixed layer $\left(-h_{m}<z<0\right)$ and in the stratified interior $\left(z<-h_{m}\right)$. Integration of the buoyancy equation (5) over depth of the upper vertically homogeneous layer results in

$$
\Psi_{r e s}\left(y,-h_{m}\right) \frac{\partial b_{m}}{\partial y}=B .
$$

In the interior, we assume that the explicit buoyancy forcing $(B)$ vanishes, and the buoyancy equation (5) reduces there to

$$
J\left(\Psi_{r e s}, \bar{b}\right)=0 \quad \text { for } \quad z<-h_{m}
$$

Using (1) and (8), we rewrite the momentum balance in terms of $\Psi_{r e s}$ as follows:

$$
\Psi_{r e s}=-\frac{\tau_{\rho}}{f}+\Psi^{*}
$$

In order to determine, for given surface boundary conditions, patterns of the buoyancy and residual circulation in the interior, MR numerically integrated the model equations (8), (9), (11) using the method of characteristics. However, we now show that the problem can be treated analytically and reduced to explicit expressions for $\bar{b}$ and $\Psi_{\text {res. }}$. The new technique reveals the dominant balances and brings more physical insight into the equilibrium dynamics of the ACC.

\section{Asymptotic analysis}

The analytical tractability is achieved by exploiting the asymptotic limit in which the residual circulation is weak relative to the time mean. Using (8) and (10), we estimate the scale $(\varepsilon)$ for the ratio of the two streamfunctions as

$$
\varepsilon \sim \frac{\Psi_{r e s}}{\bar{\Psi}} \sim \frac{B f}{b_{y} \tau_{\rho}},
$$

which thus can be thought of as a measure of the amplitude of the thermodynamic forcing relative to the mechanical forcing by winds. For the parameters representative of the Southern Ocean $\left(B \sim 2 \cdot 10^{-9} \mathrm{~m}^{2} \mathrm{~s}^{-3}\right.$ - equivalent to the heat flux of the order of $10 \mathrm{~W} / \mathrm{m}^{2}$, $\left.\tau_{\rho} \sim 2 \cdot 10^{-4} \mathrm{~m}^{2} \mathrm{~s}^{-2}, \Delta b \sim 10^{-2} \mathrm{~ms}^{-2}, L_{y} \sim 2000 \mathrm{~km}\right), \varepsilon \sim 0.2$, and therefore it is sensible to regard $\varepsilon$ as a small parameter. The solution is then obtained by expanding the interior buoyancy and residual circulation in powers of $\varepsilon$. To satisfy (10), the buoyancy forcing 
should appear at the first order $\left(B=\varepsilon B_{1}\right)$, and the wind stress $\tau_{0}$ is set at the zero order. Noting that the mean streamfunction is determined by the wind stress and therefore is independent of $\varepsilon$, we obtain

$$
\left\{\begin{array}{l}
\Psi_{r e s}=\bar{\Psi}+\Psi^{*}=\bar{\Psi}+\Psi_{0}^{*}+\varepsilon \Psi_{1}^{*}+O\left(\varepsilon^{2}\right) \\
\bar{b}=b_{0}+\varepsilon b_{1}+O\left(\varepsilon^{2}\right),
\end{array}\right.
$$

where the subscript 0 (1) denotes the zero (first) order quantities. In our model the surface buoyancy is prescribed and therefore $b_{m}(y)=\bar{b}(y, 0)$ is independent of the magnitude of buoyancy forcing, implying that

$$
b_{1}(y, 0)=0 .
$$

Next, we go on to compute the individual terms of the asymptotics in (14), thereby constructing the solution for $\bar{b}(y, z)$ and $\Psi_{\text {res }}(y, z)$.

According to (13), the expansion of $\Psi_{\text {res }}$ starts at $O(\varepsilon)$. Therefore, the leading order $(\varepsilon \rightarrow 0)$ balance of $(14)$ requires that $\Psi_{0}^{*}=-\bar{\Psi}$, regardless of a chosen eddy parameterization. This scaling argument provides a formal justification for the balance between the Eulerian and eddy-induced circulation - the key assumption of the Johnson and Bryden's (1989) model of the ACC. The buoyancy equation (11) is then trivially satisfied at zero order, whereas the eddy closure (9) reduces to

$$
\Psi_{0}^{*}=-k_{0} s_{0}^{2}=-k_{0}\left(\frac{\frac{\partial}{\partial y} b_{0}}{\frac{\partial}{\partial z} b_{0}}\right)^{2}=-\bar{\Psi}=\frac{\tau_{\rho}}{f} .
$$

Eq. (16) enables us to determine, for a given surface buoyancy distribution, the zero order interior buoyancy field as follows. Since the zero order slope

$$
s_{0}=-\sqrt{\frac{\tau_{\rho}}{\mathrm{k}_{0}|f|}}
$$

depends only on $y$, the depth of an isopycnal surface is given by an integral

$$
z=\int_{y_{0}}^{y} s_{0}\left(y^{\prime}\right) d y^{\prime},
$$

where $y_{0}$ is the location of the outcrop of this isopycnal. The derivation is simplified by introducing the indefinite integral of the slope,

$$
S(y)=\int s_{0}(y) d y
$$


which is a function only of the given wind stress. Using (19), we rephrase a statement that the zero order buoyancy is constant along the curves defined by (18) as follows:

$$
b_{0}=F[z-S(y)]
$$

where $F$, at this point, is an arbitrary function. To determine $F$, the interior buoyancy $b_{0}$ is matched, at $z \rightarrow 0$, with the surface buoyancy $b_{m}(y)$, which is assumed to be known:

$$
F[-S(y)]=b_{m}(y) \rightarrow F(r)=b_{m}\left[S^{-1}(-r)\right],
$$

an expression valid for any $r$ in the interval $0<\mathrm{r}<\max (-\mathrm{S})$. We emphasize that none of the zero order considerations here involved the buoyancy forcing.

Differentiation of (20) in $z$ shows that $\frac{\partial}{\partial z} b_{0}$ is constant along the zero order isopycnal surfaces (18). This is an expected (for our $f$-plane model) manifestation of the tendency for expulsion of the isentropic potential vorticity (PV) gradients by mesoscale eddies (Rhines and Young, 1982) which controls the structure of the leading order buoyancy field. The relatively weak $(\sim \varepsilon)$ thermodynamic forcing in our model perturbs the uniformity of the PV on the buoyancy surfaces. It is often assumed (Rhines and Young, 1982) that eddies generally flux PV down the large-scale gradient and along the isopycnals - these dynamics are represented by our model as well. Thus, inevitably, weak isentropic potential vorticity gradients result in the isopycnal fluxes of potential vorticity and other tracers. These fluxes, in turn, require presence of the residual circulation directed along the buoyancy surfaces, an effect which will appear at the next order in our expansion.

We now turn to the $O(\varepsilon)$ balance of the governing equations, which makes it possible to explicitly describe weak diabatic effects. The leading order residual streamfunction $\left(\Psi_{\text {res } 1}=\Psi_{1}^{*}\right)$ is computed using the buoyancy equation (11), whose order $O(\varepsilon)$ component is given by:

$$
J\left(\Psi_{1}^{*}, b_{0}\right)=0
$$

Eq. (22) implies that $\Psi_{1}^{*}$ and $b_{0}$ are functionally related and, by virtue of (20), that

$$
\Psi_{1}^{*}=G[z-S(y)]
$$

where $G$ is as yet an arbitrary function. Matching the interior residual streamfunction with that at the bottom of the mixed layer (10) yields:

$$
G[-S(y)]=\frac{B_{1}}{\frac{\partial}{\partial y} b_{m}},
$$

which determines the function $G$ in (23). 
Computing the modification of the interior buoyancy distribution $\left(b_{1}\right)$ due to the buoyancy forcing requires expanding the eddy closure (9) in powers of $\varepsilon$ :

$$
\Psi *=-k_{0} s^{2}=-k_{0} \frac{\left[\frac{\partial}{\partial y}\left(b_{0}+\varepsilon b_{1}+\cdots\right)\right]^{2}}{\left[\frac{\partial}{\partial z}\left(b_{0}+\varepsilon b_{1}+\cdots\right)\right]^{2}}=-k_{0} s_{0}^{2}\left(1+2 \varepsilon \frac{J\left(b_{1}, b_{0}\right)}{b_{0 y} b_{0 z}}+O\left(\varepsilon^{2}\right)\right)
$$

and the $O(\varepsilon)$ component of (25) is readily isolated thus

$$
\Psi_{1}^{*}=2 k_{0} \frac{s_{0}}{b_{0 z}} \frac{J\left(b_{1}, b_{0}\right)}{b_{0 z}} .
$$

Eq. (26) is greatly simplified by rewriting it with $b_{0}$ (rather then $\mathrm{z}$ ) as a vertical coordinate, in which case $\frac{J\left(b_{1}, b_{0}\right)}{b_{0 z}}=\left.\frac{\partial}{\partial y} b_{1}\right|_{b_{0=\text { cons }} \text {. }}$ To stress that this expression pertains to the isentropic gradients, we introduce the following notation:

$$
\frac{d}{d l}(\cdot) \equiv \frac{\partial}{\partial y}(\cdot)+s_{0} \frac{\partial}{\partial z}(\cdot)
$$

and rewrite (26) as:

$$
\Psi_{1}^{*}=2 k_{0} \frac{s_{0}}{b_{0 z}} \frac{d}{d l} b_{1}
$$

Recalling that both $\Psi_{1}^{*}$ and $b_{0 z}$ are constant at any zero order buoyancy surface, we integrate (27) in $y$, along a zero order isopycnal, from an arbitrary point in the interior $(y, z)$ to the location $\left(y_{0}, 0\right)$ where this isopycnal outcrops at the surface. Since, according to (15), $b_{1}=0$ at the surface, the integral of (27) reduces to:

$$
b_{1}\left(y, b_{0}\right)=\frac{\Psi_{1}^{*}\left(b_{0}\right) b_{0 z}\left(b_{0}\right)}{2 k_{0}} \int_{y_{0}}^{y} \frac{1}{s_{0}\left(y^{\prime}\right)} d y^{\prime} .
$$

The zero order buoyancy is known from (20) and (21), and therefore it is now straightforward to revert to the original $(y, z)$ coordinates by substituting $b_{0}(y, z)$ in (28).

\section{Explicit solutions}

To demonstrate how the foregoing balanced model can yield explicit analytical solutions, we now assume simple, easily integrable, surface conditions $\left(b_{m}, \tau_{\rho}, B\right)$, and compute 
the resulting interior fields. The estimates in MR (see their Table 1) suggest that the surface forcing fields ${ }^{2}$ can be represented by

$$
\begin{cases}b_{m}(y)=\frac{\Delta b y}{L_{y}}, & \Delta b=0.01 \mathrm{~m} / \mathrm{s}^{2}, L_{y}=2000 \mathrm{~km} \\ B=\hat{B} \sin \left(\frac{\pi y}{L_{y}}\right), & \hat{B}=2 \cdot 10^{-9} \mathrm{~m}^{2} \mathrm{~s}^{-3} \\ \tau_{\rho}=\tau_{00}\left(0.6+\frac{y}{L_{y}}\right)^{2}, & \tau_{00}=0.0001 \mathrm{~m}^{2} \mathrm{~s}^{-2} \\ f=\text { const }=-10^{-4} \mathrm{~s}^{-1}, & k_{0}=5 \cdot 10^{6} \mathrm{~m}^{2} \mathrm{~s}^{-1}\end{cases}
$$

Eqs. (17),(19) yield $S(y)=-A\left(y+0.6 L_{y}\right)^{2}$ where $A=\frac{1}{2 L_{y}} \sqrt{-\frac{\tau_{00}}{f k_{0}}}$. Inverting (21), we arrive at the expression for the zero order buoyancy:

$$
b_{0}(y, z)=\frac{\Delta b}{L_{y}}\left(\sqrt{\frac{z+A\left(y+0.6 L_{y}\right)^{2}}{A}}-0.6 L_{y}\right) .
$$

The procedure for computing the leading order residual circulation $\Psi_{\text {res }} \approx \varepsilon \Psi_{1}^{*}$ is similar. Using (24) we determine the function $G$ in (23), and thus the (leading order) residual streamfunction:

$$
\Psi_{r e s} \approx \varepsilon \Psi_{1}^{*}=\frac{\hat{B} L_{y}}{\Delta b} \sin \left[\frac{\pi}{L_{y}}\left(\sqrt{\frac{z+A\left(y+0.6 L_{y}\right)^{2}}{A}}-0.6 L_{y}\right)\right] .
$$

Finally, we estimate the changes in the interior buoyancy distribution brought by the air-sea buoyancy flux. For the wind stress given by (29), we use (30) to compute $b_{O z}$, (17) for $s_{0}$, (31) for $\Psi_{\text {res }}$, and finally reduce (28) to an explicit expression

$$
\varepsilon b_{1}=-\frac{\hat{B}}{8 k_{0} A^{2}} \frac{\sin \left[\frac{\pi}{L_{y}}\left(\sqrt{z / A+\left(y+0.6 L_{y}\right)^{2}}-0.6 L_{y}\right)\right]}{\sqrt{z / A+\left(y+0.6 L_{y}\right)^{2}}} \ln \left(\frac{0.6 L_{y}+y}{\sqrt{z / A+\left(y+0.6 L_{y}\right)^{2}}}\right) .
$$

Analytical solutions for $b=b_{0}+\varepsilon b_{1}, \Psi_{\text {res }}$ and $\varepsilon b_{1}$ given by Eqs. (30)-(32) are plotted on the right panel of Figure 2. The corresponding numerical solutions (computed as in MR) are shown on the left. Agreement between the analytical and numerical results is remarkable, especially with regard to the ability of our theory to represent the weak higher order $\left(\varepsilon b_{1}\right)$ effects. These results demonstrate that the dynamics of the ACC and its associated overturning circulation can be viewed as a leading order balance between the eddy advection and the Eulerian circulation. The diabatic buoyancy fluxes are essential in 

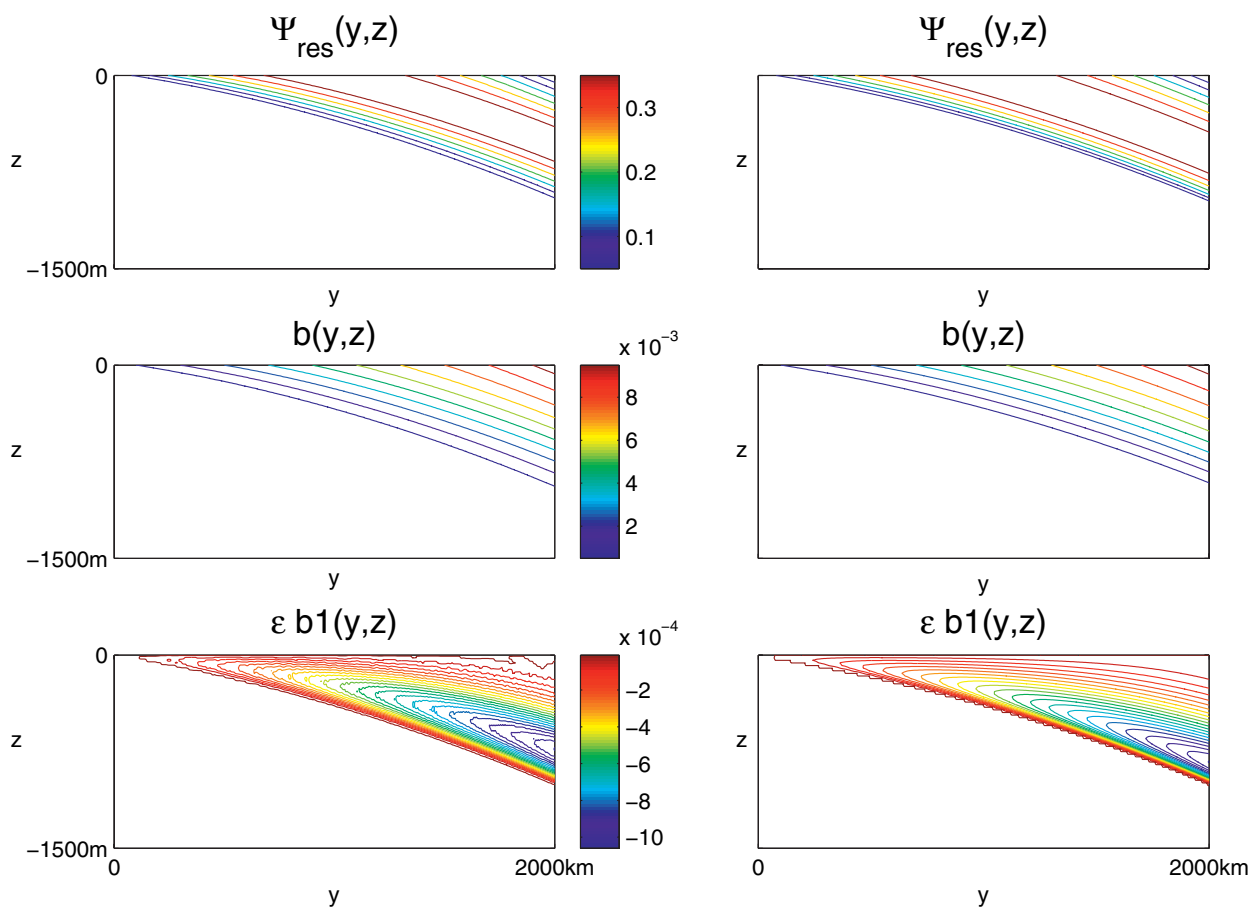

Figure 2. Comparison of the analytical solution (left) in Eqs. (30)-(32) with the corresponding numerical solution (right).

driving the secondary (residual) circulation, which is, however, steered along the pathways set by the dominant adiabatic balance.

\section{Discussion and conclusions}

This note attempts to explain the dynamics of the Antarctic Circumpolar Current by developing a balanced model based on the "weak residual circulation" approximation (2) and constructing explicit analytical solutions for the buoyancy distribution and meridional overturning circulation of the ACC. The limit considered in this study represents a regime in which the strength of the mechanical forcing by winds exceeds the strength of the buoyancy forcing. At the lowest order our expansion reflects a balance between the adiabatic eddy-induced circulation and the mean flow, a notion that is commonly used in theorizing about the dynamics of the ACC (Olbers et al., 2004). We find that this zero order balance determines gross features of the buoyancy distribution in the interior of the ACC.

At higher orders, the governing equations cannot be balanced without explicitly considering the fundamentally diabatic effects related, in our model, to the air-sea buoyancy fluxes. Making use of the dominant balance between eddies and the mean flow, we obtained closed solutions at the second order and then derived explicit expressions for 
the buoyancy and residual circulation for the idealized (but consistent with observations) forcing fields. These solutions reflect the following key characteristics of the dynamics of ACC:

i) Modification of the hydrography by the vertical mixing and air-sea fluxes $\left(\varepsilon b_{1}\right)$ is weak (Fig. 2), slightly reducing the buoyancy in the interior;

ii) Surface buoyancy flux is proportional to the strength of the residual flow, which is largely directed along the (zero order) isopycnals set up by the wind stress;

iii) Mechanics of the communication between the surface buoyancy flux and the residual circulation in the interior is related to the isentropic potential vorticity (IPV) gradients. IPV is homogeneous in the adiabatic limit $(B=0)$, but becomes slightly $(\sim \mathcal{E})$ non-uniform in the presence of buoyancy forcing, causing the down gradient eddy flux of the potential vorticity and other tracers, and thus forcing the residual flow.

It is interesting to note that while our solutions are based on the same governing equations as the fully nonlinear model in MR, their solution technique simultaneously determines both buoyancy distribution in the interior and the residual circulation, creating an impression of a strong coupling between the two. The formal asymptotic expansion, however, clarifies this issue by unambiguously separating the effects of mechanical and thermodynamic forcing (grouped together in MR). The new framework explicitly connects and reconciles the diabatic views of the Southern ocean (e.g., Speer, 2000), which emphasize the role of water mass transformation, and purely adiabatic models (e.g., Johnson and Bryden, 1989).

The finite air-sea fluxes and small-scale mixing are clearly essential for closing the buoyancy budget of the upper cell of the meridional overturning circulation in the Southern Ocean. However, it is not clear whether the role of diabatic processes in the ACC is limited to transforming the Upper Circumpolar Deep Water into the lighter Antarctic Intermediate Water, or whether they actively control the density distribution and pathways of water masses in the Southern Ocean. Our asymptotic results seem to be suggestive in this regard. The existence of closed analytical solutions in which the buoyancy fluxes $(B)$ and residual circulation $\left(\Psi_{r e s}\right)$ only appear at the second order, combined with an apparent convergence of our $\varepsilon$-expansion to the fully nonlinear solutions of the governing equations, is indicative of a largely passive role of diabatic processes for the dynamics of the Antarctic Circumpolar Current.

Of course, the simplicity of our formulation, which includes purely two-dimensional dynamics, prescribed surface buoyancy and air-sea fluxes, and exclusion of beta-effect, requires reevaluation of our ideas in more complex and realistic settings. Such studies are underway and will be reported in subsequent publications (Radko and Marshall, 2005). We note here, however, that the solutions obtained using Visbeck et al.'s (1997) closure in Section 4 and constant eddy diffusivity in the Appendix are qualitatively similar, indicating 

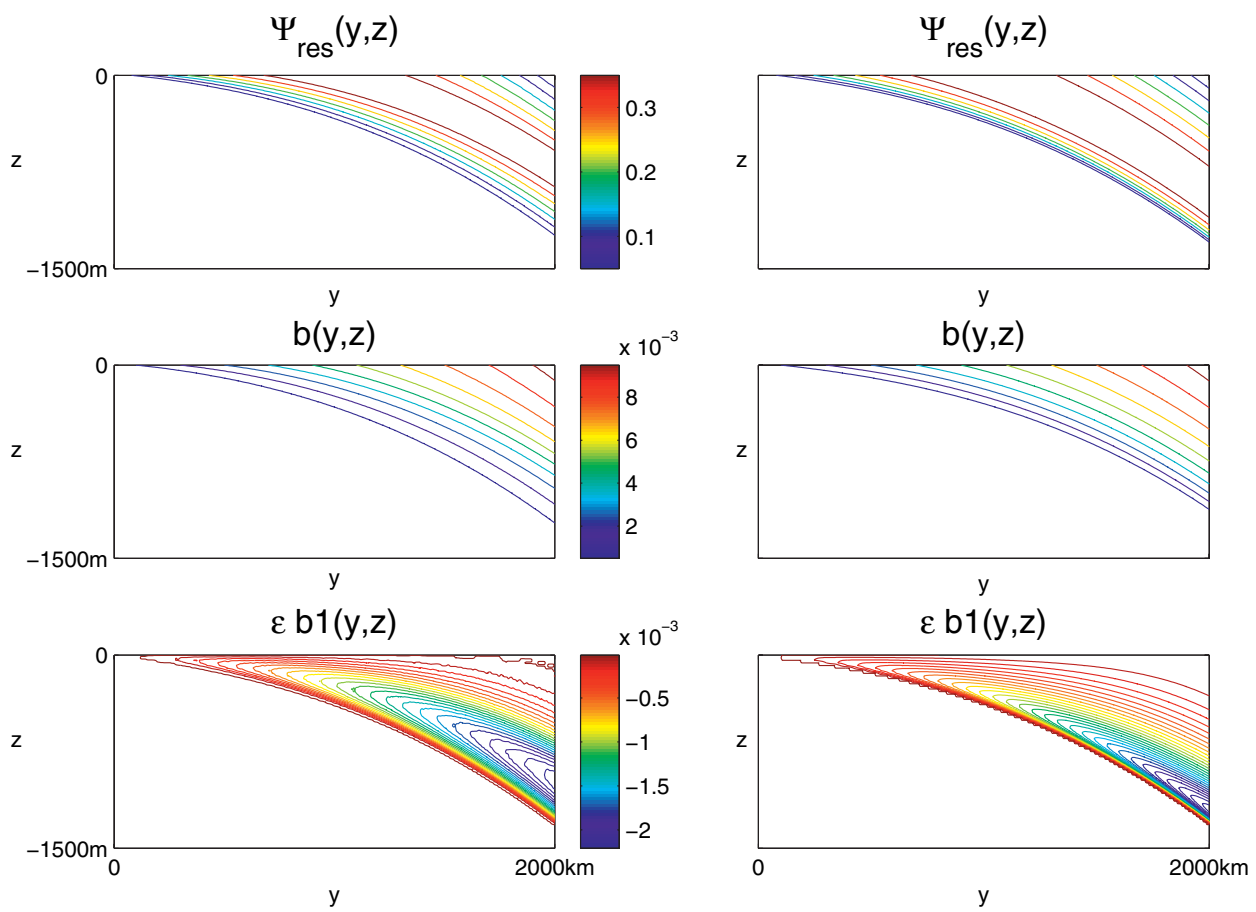

Figure 3. Comparison of the analytical solution in Eqs. (A7)-(A9) with the corresponding numerical solution.

that our theoretical results are not sensitive to a specific eddy parameterization. In both cases (Figs. 2, 3) we find a very close agreement between the asymptotic and the corresponding exact solutions for realistic values of the model parameters, which suggests that the real circumpolar current operates in the adiabatically-dominated regime and the equilibrium balances revealed by the perturbation expansion capture the essence of its dynamics.

Acknowledgments. Support of the National Science Foundation is gratefully acknowledged. The author thanks John Marshall and two reviewers for helpful comments.

\section{APPENDIX}

\section{Solutions with uniform eddy diffusivity}

Although foregoing solutions obtained using the non-uniform lateral eddy transfer coefficient $K$ are internally consistent, it is desirable to examine to what extent the results depend on the choice of a particular eddy parameterization scheme. In this appendix we consider a closure in which $K$ is set to a constant, which is widely assumed in large-scale ocean models (Gent and McWilliams, 1990). As before, we expand the residual-mean equations of motion (1), (5),(6) and (8) in powers of a small parameter $\varepsilon$ which measures 
the amplitude of the thermodynamic forcing relative to the (fixed) mechanical forcing by winds, but now the eddy-induced streamfunction is a linear function of the slope:

$$
\Psi^{*}=\frac{\overline{v^{\prime} b^{\prime}}}{\bar{b}_{z}}=K s
$$

The leading order balance of the first equation in (14) - vanishing of the Deacon cell - then requires

$$
\Psi_{0}^{*}=K s_{0}=-\bar{\Psi}=\frac{\tau_{\rho}}{f},
$$

which determines the zero order isopycnal slope

$$
s_{0}=\frac{\tau_{\rho}}{f K} .
$$

The expression for the residual streamfunction is also modified. First we expand (A1) in powers of $\varepsilon$ :

$$
\Psi^{*}=K s=-K \frac{\frac{\partial}{\partial y}\left(b_{0}+\varepsilon b_{1}+\cdots\right)}{\frac{\partial}{\partial z}\left(b_{0}+\varepsilon b_{1}+\cdots\right)}=K s_{0}\left(1+\varepsilon \frac{J\left(b_{1}, b_{0}\right)}{b_{0 y} b_{0 z}}+O\left(\varepsilon^{2}\right)\right),
$$

and then isolate the $O(\varepsilon)$ component:

$$
\Psi_{1}^{*}=-\frac{K}{b_{0 z}} \frac{J\left(b_{1}, b_{0}\right)}{b_{0 z}}=-\frac{K}{b_{0 z}} \frac{d b_{1}}{d l} .
$$

Since $\Psi_{1}^{*}$ and $b_{0 z}$ are constant at any zero order isopycnal, integration of (A5) from the interior to the surface along isopycnals yields an expression for the modification of buoyancy distribution due to the air-sea fluxes:

$$
b_{1}(y, z)=-\frac{\Psi_{1}^{*} b_{0 z}}{K}\left(y-y_{0}\right) .
$$

We now go on to derive explicit solutions for buoyancy and residual circulation for the same forcing functions (29) as we used in Section 4. Based on the estimates in Karsten and Marshall (2002), we use $K=2000 \mathrm{~m}^{2} / \mathrm{s}$, which is comparable to the values of $K$ in the foregoing (Sec. 4) solutions, but larger than the canonical $1000 \mathrm{~m}^{2} / s$ used in coarseresolution numerical models. For the zero order slope given by (A3), Eq. (19) yields $S(y)=\frac{\tau_{00} L_{y}}{3 K f}\left(0.6+\frac{y}{L_{y}}\right)$. Thus, inverting (21), we determine the zero order buoyancy:

$$
b_{0}(y, z)=\Delta b m, \quad \text { where } \quad m=\sqrt[3]{\left(0.6+\frac{y}{L_{y}}\right)^{3}-\frac{3 K f z}{\tau_{00} L_{y}}}-0.6
$$


Similarly, using Eqs. (23), (24), we compute the (leading order) residual streamfunction:

$$
\Psi_{r e s} \approx \varepsilon \Psi_{1}^{*}=\frac{\hat{B} L_{y}}{\Delta b} \sin (\pi m),
$$

and (A6) reduces to an expression for $\varepsilon b_{1}$ :

$$
\varepsilon b_{1}=\frac{f \hat{B} \sin (\pi m)}{\tau_{00}(m+0.6)^{2}}\left(y-L_{y} m\right) .
$$

These analytical solutions are plotted in Figure 3 on the right, along with their numerical counterparts (computed using the technique developed in MR) on the left. As previously (Sec. 4), we find a very close agreement between the asymptotic and exact solutions for realistic values of the model parameters, which is indicative of a robust and closureindependent character of the balanced weak residual circulation theory developed herein.

\section{REFERENCES}

Andrews, D. G. and M. E. McIntyre. 1976. Planetary waves in horizontal and vertical shear: the generalized Eliassen-Palm flux and the mean zonal acceleration. J. Atmos. Sci., 33, 2031-2049.

Bryden, H. L. and S. A. Cunningham. 2003. How wind-forcing and air-sea heat exchange determine the meridional temperature gradient and stratification for the Antarctic Circumpolar Current. J. Geophys. Res., 108(C8), 3275. doi:10.1029/2001JC001296.

Cenedese, C., J. Marshall and J. A. Whitehead. 2004. A laboratory model of thermocline depth and exchange fluxes across circumpolar fronts. J. Phys. Oceanogr., 34, 656-667.

Danabasoglu, G., J. C. McWilliams and P. Gent. 1994. The role of mesoscale tracer transport in the global ocean circulation. Science, 264, 1123-1126.

Doos, K. 1994. Semianalytical simulation of the meridional cells in the Southern Ocean. J. Phys. Oceanogr., 24, 1281-1293.

Doos, K. and D. Webb. 1994. The Deacon cell and other meridional cells of the Southern Ocean. J. Phys. Oceanogr., 24, 429-442.

Gent, P. and J. McWilliams. 1990. Isopycnal mixing in ocean circulation models. J. Phys. Oceanogr., $20,150-155$.

Gnanadesikan, A. and R. W. Hallberg. 2000. The relationship of the Circumpolar Current to Southern Hemisphere winds in coarse-resolution ocean models, J. Phys. Oceanogr., 30, 20132034.

Hallberg, R. W. and A. Gnanadesikan. 2001. An exploration of the role of transient eddies in determining the transport of a zonally reentrant current, J. Phys. Oceanogr., 31, 3312-3330.

Held, I. M. and T. Schneider. 1999. The surface branch of the zonally averaged mass transport circulation of the troposphere. J. Atmos. Sci., 56, 1688-1697.

Johnson, G. C. and H. L. Bryden. 1989. On the size of the Antarctic Circumpolar Current. Deep-Sea Res., 36, 39-53.

Karsten, R., H. Jones and J. Marshall. 2002. The role of eddy transfer in setting the stratification and transport of a circumpolar current. J. Phys. Oceanogr., 32, 39-54.

Karsten, R. and J. Marshall. 2002. Constructing the residual circulation of the ACC from the observations. J. Phys. Oceanogr., 32, 3315-3327.

Marshall, J. and T. Radko. 2003. Residual-mean solutions for the Antarctic Circumpolar Current and its associated overturning circulation. J. Phys. Oceanogr., 33, 2341-2354. 
Olbers, D., D. Borowski, C. Volker and J. O. Wolff. 2004. The dynamical balance, transport and circulation of the Antarctic Circumpolar Current. Antarctic Science, 16, 439-470.

Radko, T. and J. Marshall. 2005. The Antarctic Circumpolar Current in three dimensions. J. Phys. Oceanogr., (in press).

Rhines P. B. and W. R. Young. 1982. Homogenization of potential vorticity in planetary gyres. J. Fluid Mech., 122, 347-367.

Speer, K. G., B. Sloyan and S. R. Rintoul. 2000. The diabatic Deacon Cell. J. Phys. Oceanogr., 30, 3212-3222.

Visbeck, M., J. Marshall, T. Haine and M. Spall. 1997. On the specification of eddy transfer coefficients in coarse-resolution ocean circulation models. J. Phys. Oceanogr., 27, 381-402.

Received: 7 September, 2004; revised: 29 June, 2005. 